\title{
Review of "Users' guide to Knowledge management with concept maps and mind maps: what are these and a proposal of a workflow"
}

\author{
Giorgio Bedog $\mathrm{ni}^{1}$ \\ 1 Fondazione Italiana Fegato ONLUS
}

I found this article of great interest for two reasons. The first reason is that it tries to put together the best of two tools to manage knowledge, i.e. concept maps and mind maps, which are often simply perceived as conflicting and tend to polarize users. The second reason is that this is an article that can benefit most Qeios readers independently from their research background. Stimulated by this article, I am currently studying reference \#4. I hope to see more on this topic. 